Relations industrielles

Industrial Relations

\title{
Un commentaire européen
}

\section{Dimitri Weiss}

Volume 44, numéro 4, 1989

URI : https://id.erudit.org/iderudit/050541ar

DOI : https://doi.org/10.7202/050541ar

Aller au sommaire du numéro

\section{Éditeur(s)}

Département des relations industrielles de l'Université Laval

ISSN

0034-379X (imprimé)

1703-8138 (numérique)

Découvrir la revue

\section{Citer cet article}

Weiss, D. (1989). Un commentaire européen. Relations industrielles / Industrial

Relations, 44(4), 914-920. https://doi.org/10.7202/050541ar
Résumé de l'article

Un commentaire européen
Tous droits réservés (C) Département des relations industrielles de l'Université Laval, 1989
Ce document est protégé par la loi sur le droit d'auteur. L’utilisation des services d'Érudit (y compris la reproduction) est assujettie à sa politique d'utilisation que vous pouvez consulter en ligne.

https://apropos.erudit.org/fr/usagers/politique-dutilisation/ 
ones will permit the academic research field to better serve the needs of the practitioners as well as enrich the theoretical field. The efforts of the past in this endeavor must be rejuvenated by new ones. More profound and encompassing analyses are needed of the Canadian system to permit its participants and students better to understand and appreciate its distinctive characteristics, innovations and trends.

\section{Un commentaire européen}

\section{Dimitri Weiss}

Il n'est pas sans intérêt de noter que la parution de ce volume à l'occasion du XXV' anniversaire de l'Association canadienne des relations industrielles, intervient juste trente ans après la publication de Industrial Relations Systems par John T. Dunlop, comme pour faire le point de l'évolution des idées au bout de trois décennies que certains avaient, pratiquement, perçues comme un règne sans partage.

Il est certain que $L$ 'état de la discipline en relations industrielles au Canada est un ouvrage qui trouvera sa place sur la même étagère que le monumental Dictionnaire canadien des relations du travail de Gérard Dion - objet, justifié, de la première ligne et de la première référence, page 1 du chapitre $\mathrm{I}$ - au titre des contributions majeures du Canada à l'intelligence de notre domaine commun. Car sa portée, à l'instar de l'oeuvre de Dion, dépasse visiblement les frontières du pays, fussent-elles impressionnantes, pour investir l'Amérique du Nord dans son ensemble et nous fournir des enseignements et des observations utiles pour notre propre situation - je parle en tant qu'européen -, dont l'évaluation reste, en partie, tributaire des pensées du Nouveau Monde.

Notre domaine commun, ai-je dit, ne signifie pas uniquement celui des Américains, des Européens et des autres, mais aussi - et surtout - de tous ceux qui, à partir des domaines disciplinaires d'apport traditionnels - économie, droit, sociologie, psychologie, histoire, sciences politiques - à ceux de plus récente pénétration dans nos préoccupations majeures - le management, la gestion - et aux chercheurs qui en ont une approche transdisciplinaire ou transversale, se sentent partie prenante d'un champ, d'une discipline ou d'une multidiscipline, dont ils se réclament avec une égale force.

Dans la cinquième édition des Relations du travail: employeurs, personnel, syndicats, État, (Dunod, 1984 (1983), p. 10), j'avais pour ma part observé combien l'exigence d'un échange entre optiques et méthodologies diverses avait été accompagnée d'une ouverture de chacune des disciplines composantes vers d'autres, avec lesquelles s'établissent des liaisons et des

* WEISS, Dimitri, professeur, Université de Paris, Panthéon-Sorbonne, Institut d'Administration des Entreprises. 
frontières mobiles. Ce qui était autrefois un paysage d'îles isolées, avec une communication souvent aléatoire, apparaît aujourd'hui comme un archipel dont chaque unité, tout en conservant son individualité, participe, en même temps, à un patrimoine commun. Aussi parlerait-on du vaste domaine des relations du travail, et du champ plus étendu encore des relations industrielles, non plus comme d'une somme de disciplines individuelles, mais comme d'une synthèse permanente, d'une dialectique ininterrompue, d'une synergie intellectuelle dépassant les optiques disciplinaires et nationales en vue d'une intégration de la recherche, elle-même en expansion continue.

En d'autres termes, ajoutais-je, le savoir dans le domaine des relations industrielles n'est pas, ou ne devrait pas être, le total d'une série d'additions, mais le résultat, fût-il parfois douteux ou discutable, d'une série d'articulations ou d'interférences entre différents facteurs autonomes. Les distinctions et les oppositions rigides, tout ce qui isole les matières d'apport en fortins sans portes ni fenêtres, apparaissent de plus en plus inadéquates et arbitraires. Ce savoir n'est, donc, pas une «somme» coûteuse dans un inventaire précaire (et poussiéreux), mais un entrelacement de routes à l'horizon quelquefois lointain.

Hébert, Jain et Meltz posent bien le problème dès le premier chapitre, en choisissant de définir les «relations industrielles» comme domaine global des relations du travail (rapports collectifs), des relations avec le personnel - que j'appelle, pour ma part, à l'italienne, «relations internes» - et qui sont justiciables de la gestion des ressources humaines - et des relations d'emploi, les relations industrielles «incluant tous les aspects du travail et les multiples rapports ou relations qui en découlent au lieu de travail». $\tilde{A}$ quoi j'ajouterais, en suivant en cela G. Caire, «à l'occasion ou à propos du travail».

Les trois directeurs de l'ouvrage n'hésitent pas, en même temps, de rendre compte du débat, qu'ils jugent pérenne, autour de la «discipline» des relations industrielles, naissante ou en voie de développement, ou autour de l'«art», sans prendre, naturellement, de position, attitude réitérée dans la conclusion: «le présent ouvrage ne peut conclure si les relations industrielles sont une discipline ou un sujet d'étude ou bien même un art». Mais ils ont pris, néanmoins un risque (langagier) d'engendrer un malentendu en attribuart au livre le titre «l'état de la discipline». Pourquoi pas «l'état de l'art»? Une troisième expression eût été la bienvenue, mais laquelle? L'état du «domaine»? L'état de la (multi)spécialité? Le cours de perfectionnement en relations industrielles et du travail (titre probablement inspiré de celui de l'École de Cornell) de l'Université de Bologne vient, après une lutte de dix ans, d'acquérir le statut d'École de spécialisation en la matière. Mais, là aussi, le débat risque de rester durable...

Alton W.J. Craig, dont les directeurs de l'ouvrage signalent pertinemment sa contribution à l'approche systémique quelques années après Dunlop - son chapitre en est une illustration - se prononce sur les principaux aspects des relations industrielles au Canada en faveur d'une étude multidisciplinaire, seule à pouvoir éclairer les multiples facettes desdites relations, dont il donne, d'ailleurs, une définition compréhensive. 
Morley Gunderson, dans sa contribution «Économie du travail et relations industrielles»), appelle à une interfécondation entre les deux composantes du titre - d'ailleurs chacun difficilement définissable d'une manière univoque - et il présente le premier comme étant une partie intégrante du système engendré par le second. Il ne méconnait pas les accrocs à une communication interdisciplinaire dûs à la mathématisation croissante de l'économie, mais il n'en relève pas moins les possibilités et les exemples de synthèse possible (grèves, pratiques de gestion des ressources humaines retraites, pensions, systèmes de rémunérations, heures et durée du travail -, impact du syndicalisme), ce qui ancre solidement ses arguments dans le champ empirique.

Se référant particulièrement à la réalité juridique québécoise, Pierre Verge étudie, de son côté, le rapport entre le droit et les relations du travail - tandis que Bernard Adell s'attache, lui, au droit et aux relations du travail dans les juridictions canadiennes de Common Law - , en observant combien la pratique de ces relations s'était en partie «judiciarisée» (ce que Côté, Lemelin et Toulouse signalent, dans la contribution suivante, comme une évolution majeure des années 1980), et comment le droit du travail y «a établi une emprise virtuelle quasi totale». (On a attribué, a contrario, au cours de cette décennie, à la prédominance historique du droit dans certains pays européens, vue comme génératrice d'inertie, le retard pris dans la gestion des ressources humaines, la forte réaction d'individualisation de la gestion et des relations et la marginalisation des rapports syndicaux.)

Mais Verge, en évoquant leur interaction, n'insiste pas moins sur les questions: comment la réalité du travail peut modeler le droit, comment elle continuera de l'influencer et, enfin, comment le juriste devrait «idéalement, se doubler d'un sociologue, d'un économiste, d'un psychologue, d'un historien», ce qui "comporte, au minimum, une connaissance élémentaire de l'apport et de la méthodologie de ces autres sciences du travail» et le profit qu'il peut en tirer.

Si les rapports interdisciplinaires étudiés jusqu'ici furent traditionnellement évoqués, sinon étudiés, dans l'évolution des relations industrielles, les liens entre le management et celles-ci, expliqués judicieusement et amplement par Marcel Côté, Maurice Lemelin et Jean-Marie Toulouse, présentent un caractère de relative nouveauté. Les auteurs montrent comment les recherches - et publications - en management et en relations du travail prouvèrent une ignorance réciproque, ce qui vaut, d'ailleurs, des deux côtés de l'Atlantique.

Le management, disent-ils, en ajoutant qu'il «faut faire ici exception de la tradition française, ne s'est pas inspiré des disciplines juridiques pour développer son savoir». Sont-ils aussi convaincus pour des pays au background culturel aussi différent que l'Italie et l'Allemagne? Ce que je constate, en tout cas, aujourd'hui, chez certains parmi mes propres étudiants de $3^{\mathrm{e}}$ cycle en relations industrielles, responsables de ressources humaines dans leurs entreprises et managers divers, c'est un désintérêt croissant pour la chose juridique, allant parfois jusqu'à une crasse ignorance: on est passé d'un extrême à l'autre. 
Il est vrai qu'on assiste, en ce moment, à l'accentuation du rapport avec le salarié individuel, au-delà de ce qui est prévu par la négociation collective et la législation du travail, et que ce rapport ne s'épuise pas dans des aspects contractuels formels ou dans une froide énumération de droits et de devoirs, car le changement et la qualité du travail, du produit ou du service, et l'excellence des comportements trouvent leur source plutôt dans une mobilisation d'énergies que dans un aperçu intellectuel de règles traduisant des exigences et des obligations. Mais le droit des sociétés, le droit économique de l'entreprise, le droit des affaires - et je signale ici les éditions successives du livre sur le sujet de Pierre Montreuil (un «transdisciplinaire» par excellence pour qui les relations industrielles n'ont aucun secret), paru chez Gaëtan Morin - et, surtout, la gestion juridique du travail et de l'emploi, des ressources et des relations, ne peuvent être absents de la préparation d'un cadre responsable d'entreprise, fût-il en relations industrielles, et cela me semble bien transparaître des observations de nos trois auteurs.

Ils posent, pour la première fois, à ma connaissance, en français, sur le fond, les rapports ambigus entre les deux termes du débat, qu'Hébert, Jain et Melz soulèvent en évoquant des "champs d'études» et que Côté, Lemelin et Toulouse définissent comme étant "deux solitudes», qui aurait intérêt, chacune, à intégrer les connaissances de l'autre.

Et, pour ce faire, ils décrivent bien les écoles de pensée en management et les liens entre celui-ci et les relations industrielles à travers la notion de stratégie. (Pour ce qui est de l'utilisation de cette notion en relations industrielles, je rappelle, notamment, l'observation critique de David Lewin dans «Industrial Relations as a Strategic Variable», Human Resources and the Performance of the Firm, IRRA, 1987).

À la clé, une idée simple: les relations industrielles d'une firme sont, aujourd'hui, beaucoup plus déterminée par les stratégies de ladite firme que par le système national de relations du travail. D'où le constat, approprié, des auteurs sur la secousse enregistrée dans les rapports entre les acteurs du système et l'émergence d'une gestion des ressources humaines qui, dans sa centralité et le lien ressources-relations-résultat est en train de changer les données et les règles du jeu. (Je signale ici l'intéressante communication de Michel Audet et de Laurent Bélanger au $1^{\text {er }}$ Congrès des relations industrielles des Amériques, sur la «Gestion des organisations dans les Amériques et leur impact sur les relations industrielles», avec comme sujet d'intervention «Environnement turbulent, nouveaux paramètres organisationnels et impact sur les relations industrielles».)

Les auteurs, ai-je dit, accordent une grande importance à «la notion de stratégie d'entreprise [qui] fournit un angle privilégié pour comprendre les rapports entre le management et les relations industrielles». Dans un utile développement, au cours duquel ils passent en revue auteurs, courants et modèles, ils notent, particulièrement, le fait que les «actions posées dans ce domaine des relations industrielles découlent et sont dépendantes des choix stratégiques [...], de l'orientation stratégique donnée par l'entreprise [...et que] «la stratégie détermine les enjeux des relations industrielles», et concluent sur l'engagement graduel des salariés dans les décisions stratégiques de l'entreprise et, de là, sur le rôle croissant des ressources humaines dans la 
détermination des stratégies de relations industrielles, «les principales actions stratégiques des gestionnaires étant celles qui sont reliées aux ressources humaines».

Je voudrais insister ici, un peu plus, sur la place importante que me semble revêtir, quant aux choix stratégiques comme élément moteur du système, le livre de Kochan, Katz et McKersie intitulé The Transformation of American Industrial Relations, présent dans la bibliographie, très complète. La théorie des choix stratégiques - Isabel da Costa l'a bien observé - intègre l'institutionnalisation des sciences du comportement dans la pratique des relations industrielles aux États-Unis, et l'apport de l'école du MIT apparaît comme la contribution majeure au débat en cours par sa révision théorique du modèle de Dunlop, auquel je faisais allusion au début de cette recension: «Les pratiques et les résultats des relations industrielles sont façonnés par les interactions des forces de l'environnement avec les choix stratégiques et les valeurs des employeurs américains, des leaders syndicaux, des salariés et des décideurs en matière de politique publique». Cela a-t-il (eu) une influence sur «l'état de la discipline» au Canada?

Ajoutons que Côté, Lemelin et Toulouse notent, à l'instar d'Alton Craig, le manque d'études dans le domaine des relations industrielles concernant les employeurs, ce qui n'est pas uniquement, hélas, le cas du Canada.

Les directeurs de l'ouvrage ont laissé, sagement, au lecteur le soin «de décider pour lui-même jusqu'à quel point les sciences de l'administration et le comportement organisationnel se recouvrent l'un l'autre, en spécifiant, plus haut, «domaine des sciences de l'administration ou du management».

C'est bien de management dont ont traité Côté, Lemelin et Toulouse, et il ne s'agit pas, à mon avis, d'un terme identique ou équivalent à celui des sciences de l'administration (ou de gestion). L'Academy of Management, aux États-Unis, n'est pas une Academy of Business Administration... Aussi était-il normal que, dans la succession logique envisagée par Hébert, Jain et Meltz, John Kervin montre le rôle actuel des sciences du comportement sociologie, psychologie - dans les relations industrielles.

Déjà Alton Craig appelait à combler le fossé entre les chercheurs en sciences du comportement et les spécialistes des relations industrielles, les programmes de recherche en relations industrielles devant comporter une meilleure intégration des résultats de la recherche en comportement organisationnel, de la même manière que les behavioristes auraint intérêt à mieux situer et comprendre le rôle des rapports collectifs et, en particulier, des syndicats.

Kervin définit les sous-champs de la sociologie et de la psychologie où l'on trouve, dit-il, les contributions les plus fécondes aux relations industrielles. D'abord, la sociologie industrielle, qui examine les rapports découlant du travail et de l'organisation industrielle. Ensuite, la sociologie du travail, laquelle s'intéresse aux occupations, aux marchés du travail, à 
l'environnement et aux groupes de travail. Enfin, la psychologie industrielle, qui s'attache aux habitudes et aux comportements des individus dans le contexte industriel, et dont le comportement organisationnel constitue l'aspect appliqué, quid, alors, de la psychologie du travail, des liens entre développement des organisations et management stratégique, etc.?

L'auteur note qu'à l'heure actuelle, il se fait plus de travaux dans les sciences «du comportement en Grande-Bretagne, qu'aux États-Unis ou au Canada». Il est intéressant de noter, à cet égard, que lors du colloque italobritannique de sociologie du travail qui eut lieu à Bologne en 1988, ayant pour thème «Organisation du travail, nouvelles technologies et relations industrielles: les cas italien et anglais"), Duncan Gallie, de l'Université d'Oxford, évoquait, dans son rapport intitulé De la sociologie industrielle à la sociologie du travail: un panorama de la recherche anglaise à partir des années 60 , le lien entre emploi et structure des relations familiales et la manière dont les dynamiques de la famille structurent la nature et le flux de l'offre de travail. Il concluait en observant que «la discussion émergente concernant le rôle de la famille a ouvert encore plus l'horizon, en liant l'emploi à une sphère large d'activités de travail, et en complétant ainsi le déplacement de la «sociologie industrielle» initiale à celle que nous pouvons effectivement appeler aujourd'hui «sociologie du travail». Ce qui semblait être de plus en plus évident était l'interdépendance entre l'organisation du travail dans le secteur de l'emploi formel, l'organisation du travail non formelle et domestique, et la nature des réseaux sociaux locaux [...]».

Kervin compare les sciences du comportement à l'économique, en mettant en relief les différences entre les unités d'analyse, les postulats fondamentaux et, surtout, l'accent mis sur les processus plutôt que sur les résultats, dont les exemples les plus éclairants se trouvent dans les recherches sur la négociation collective. Celle-ci fait l'objet, après «les syndicats», «les attitudes vis-à-vis des syndicats», et la «technologie et [les] changements technologiques», d'une section mettant en évidence le rôle particulier des psychologues. Et si l'auteur a raison de dire qu'il s'agit là plutôt d'une préoccupation nord-américaine, sauf exceptions parmi les behavioristes anglais et européens, je signale parmi ces derniers les italiens Enzo Spaltro et Marisa Cengarle (voir Weiss, La fonction Ressources humaines, Paris, les Éditions d'Organisation, 1988).

Les nombreuses pages que Kervin consacre au sujet m'ont vivement intéressé (dynamique - études de laboratoire, modèles, autres études problèmes méthodologiques, résultats, médiation), au même titre que celles vouées par Alton Craig et par Bernard Adell au même processus de négociation, dans des optiques différentes, ce dernier y ajoutant des considérations, tout aussi utiles, sur le droit à la négociation collective. Et on peut en dire autant du conflit industriel et de sa traduction manifeste, la grève. Le fait que ces concepts traversent les pages de cet ouvrage et les approches disciplinaires académiques montrent la fertilité d'un traitement croisé.

Il revenait aux historiens, Desmond Morton et James Thwaites d'écrire le dernier chapitre de cet ouvrage rare, dense, bienvenu et complet, clairement écrit, et auquel ce compte-rendu partiel, partial et sélectif ne rend qu'insuffisamment justice. 
Tous les auteurs ont apporté une contribution substantielle, et grâces leur soient rendues pour avoir si bien su stimuler la discussion et servi le domaine de nos préoccupations communes. Une gratitude particulière aux trois directeurs de l'ouvrage, avec une pensée particulière pour Gérard Hébert, coordonnateur et traducteur de l'édition française.

J'ajoute que différents index complètent le livre: des auteurs, des lois, des décisions judiciaires, des disciplines, des matières.

Ouvrage à lire nécessairement.

\section{INDUSTRIAL RELATIONS JOURNAL}

\section{Edited by Brian Towers}

The Industrial Relations Journal, established in 1970, is a leading UK-based journal with an international flavour, which aims to monitor, report and predict significant developments in industrial relations for practitioners, policy-makers and academics. Articles in the Industrial Relations Journal have included studies of trade unions in modern industrial society, the industrial relations practices of foreign-owned multinationals, and the decline of mass production and union survival in the USA. The journal has recently launched a new feature on Trends and Developments in Industrial Relations and the Law. The annual subscription includes four issues of IRJ plus two companion issues of New Technology, Work and Employment, edited by Colin Gill.

Recent articles include:

Trends and developments in industrial relations law: The Employment Act 1988 KARL MACKIE

Japanisation: a lack of chemical reaction MICK MARCHINGTON

Working-time reduction: a survey of the Australian experience JOHN MANGAN and JOHN STEINKE

Industrial Relations Journal is published in Spring, Summer, Autumn and Winter plus free subscription to New Technology, Work \& Employment (published Spring and Autumn)

Subscription Rates Volume 20, 1989

Individuals: $\$ 50.00$ (UK) $£ 59.50$ (overseas) US $\$ 99.00$ (N. America)

Institutions: $\Sigma 65.50$ (UK) 278.50 (overseas) US $\$ 129.50$ (N. America)

\section{Basil Blackwell • Journals Marketing Manager,}

108 Cowley Road, Oxford OX4 1JF UK or, Journals Marketing Manager, 432 Park Avenue South, NY 10016, USA. 